\title{
Mesenchymal Stem Cells-derived Exosomal miR- 148a-3p Alleviates Atrial Fibrosis and Vulnerability to Atrial Fibrillation Through Targeting ALK5/Smad2 Axis
}

\section{Daoliang Zhang}

Shanghai Jiao Tong University Affiliated Chest Hospital

\section{Qian Wang}

Shanghai Jiaotong University School of Medicine Xinhua Hospital

Jie Liu

Weifang Medical University

\section{Guangchen Zou}

Danbury Hospital

Shili Jiang

Laiyang Health School

Wenbo Liu

Weifang Medical University

Huirong Han

Weifang University

Liye Zhang

ShanghaiTech University

\section{Rui Zhang}

Weifang Medical University

Qijuan Sun ( $\nabla$ sunqijuan_1983@sina.com )

Weifang Medical University https://orcid.org/0000-0001-6352-7895

\section{Research}

Keywords: Mesenchymal stem cells, Exosome, MiRNA-148a-3p, Atrial fibrosis, Atrial fibrillation

Posted Date: September 1st, 2021

DOl: https://doi.org/10.21203/rs.3.rs-847266/v1

License: (c) (i) This work is licensed under a Creative Commons Attribution 4.0 International License. Read Full License 
Page $2 / 25$ 


\section{Abstract}

Aims: Atrial fibrosis is the hallmark of atrial fibrillation (AF). Accumulating evidence have shown that mesenchymal stem cells (MSCs) can alleviate fibrosis in diverse organs. However, the role of MSCsderived exosome (MSCs-Exo) in the pathogenesis of atrial fibrosis and vulnerability to AF was unknown.

Methods: Exosomes were isolated from cultured MSCs. Transmission electron microscopy and western blot were used to examine the purity of MSCs-Exo. 8-week-old BALB/c mice were randomized into sham group, angiotensin-II (Ang-II) group, and Ang-II+MSCs-Exo group. A mini-pump was implanted for subcutaneous infusion of Ang-II for 4 weeks to induce atrial fibrosis. In the Ang-II+MSCs-Exo group, $300 \mu \mathrm{g} / 150 \mu \mathrm{l} \mathrm{MSCs-Exo}$ were injected into tail veins twice a week after establishing the animal model. Echocardiography was used to evaluate heart structure and function. Intracardiac electric stimuli was used to analyze AF inducibility. Masson staining was used to evaluate the degree of atrial fibrosis. Ang-IIinduced proliferation, migration, phenotypic switching and the capacity of extracellular matrix synthesis were examined after MSCs-Exo preconditioning. We reanalyzed two public expression datasets of MSCsExo and selected a few most highly expressed microRNAs. After literature search and experimental validation, we narrowed down our candidates to miR-148a-3p. Then, we predicted the potential target genes of miR-148a-3p, and determined $A L K 5$ and SMAD2 as the targets of miR-148a-3p. Western blot was used to quantify Ang-II-induced protein expressions of TGF- $\beta 1 /$ ALK5/SMAD2 pathway in fibroblasts after exposure to miR-148a-3p-rich MSCs-Exo.

Results: Compared with mice in Ang-II group, mice in Ang-II+MSCs-Exo group showed less atrial enlargement, atrial fibrosis, a lower AF inducibility and AF duration. Ang-II-induced proliferation, migration, phenotypic switching, and capacity of extracellular matrix synthesis were ameliorated in fibroblasts after co-incubation with MSCs-Exo. After exposure to miR-148a-3p inhibitor, MSCs derived Exosomes (MSCs148a-3p inhibitor-Exo) can significantly reverse above phenomenon. As the targets of miR-148a-3p, the expressions of ALK5 and Smad2 were lower in Ang-II+MSCs-Exo group compared with the Ang-II group. Furthermore, MSCs-Exo treatment inhibited Ang-II-induced activation of TGF- $\beta 1 /$ ALK5/Smad2 pathway, and MSCs-148a-3p inhibitor -Exo reverse above phenomenon.

Conclusions: MSCs-Exo can alleviate Ang-II induced atrial fibrosis and AF vulnerability through transferring miRNA-148a-3p to inhibit the activation of TGF-31/ALK5/Smad2 pathway, providing a new avenue to AF treatment.

\section{Background}

Atrial fibrillation (AF) is most common arrhythmia in clinical practice, with increasing incidence with aging and accompanied heart diseases [1]. It is a major risk factor for ischemic stroke, heart failure, and all-cause mortality, bringing heavy financial burden to society and family [2]. Structural remodeling was observed in both experimental and clinical AF paradigms, and it was a vital feature of AF substrate. Atrial fibrosis is the hallmark of structural remodeling. So far, evidence is mounting that atrial fibrosis is key in 
promoting the occurrence and maintenance of AF. Greater atrial fibrosis is more likely observed in patients with persistent $\mathrm{AF}$ and is associated with more recurrences after $\mathrm{AF}$ catheter ablation. Therefore, targeting atrial fibrosis is considered as an important strategy to prevent and treat AF.

Mesenchymal stem cells (MSCs) derived from bone marrow are an important source of stem cells. MSCs have been observed to confer functional and structural benefits in diseased heart, including enhanced angiogenesis, reduction of infarct size, improvement of cardiac function, reduction of fibrosis area, and reduction of inflammatory response [3, 4]. Although MSCs transplantation has achieved good results, the transplanted stem cells have a low survival rate and integration rate [5]. Furthermore, MSCs are difficult to differentiate into cardiac cells, which limit the roles of MSCs to improve cardiac function. Accumulating evidence supports the views that MSCs act in a paracrine manner. The biological factors including exosomes and soluble factors derived from the conditioned medium of MSCs cultures are explored extensively. Exosomes are small vesicles, 30 to $100 \mathrm{~nm}$ in diameter. Several studies have claimed that MSCs-Exo have functions similar to those of MSCs, and increasing attentions are being paid to the therapeutic potentials of MSCs-Exo. MSCs-Exo fuse with remote cells delivering a cargo of functional proteins including mRNAs, microRNA (miRNAs) and IncRNAs that can modify the phenotype of the target cells [6].

MiRNAs acted as key regulators of cell-cell communication and paracrine signaling mediators during physiological and pathological processes. MSCs-Exo-derived miRNA showed beneficial effects on cardiac ischemia-reperfusion injury ${ }^{4}$, post-myocardial infarction cardiac injury [7, 8], and cardiac hypertrophy [9]. However, little is known about the effect of MSCs-Exo or MSCs-Exo-derived miRNA on atrial fibrosis and vulnerability to $A F$, and the underlying mechanism is still incomplete. In this study, we demonstrated the effect of MSCs-Exo on atrial fibrosis and AF vulnerability, which is mainly through passing on exosomal miR-148a-3p in order to inhibit the activation of TGF- $\beta 1 / A L K 5 / S m a d 2$ pathway.

\section{Methods}

\section{Ethical statement}

All experimental procedures were performed with the approval of the Ethics Committee as well as the Animal Care and Use Committee of Shanghai Chest Hospital affiliated to Shanghai Jiao Tong University.

\section{Bone marrow-derived MSCs-Exo isolation and characterization}

Mouse bone marrow-derived MSCs were purchased in Cyagen Biosciences Inc. (Santa Clara, CA, US). When MSCs reached $70 \%-80 \%$ confluency, culture medium was replaced by that containing $5 \%$ exosomefree fetal bovine serum and then MSCs were cultured for $48 \mathrm{~h}$. MSCs-Exo were isolated using differential centrifugation using ExoEasy Maxi Kit (QIAGEN, Germantown, MD, US). The morphology of MSCs-Exo was observed using transmission electron microscope. After isolation, $10 \mu \mathrm{l}$ MSCs-Exo were diluted in 1 $\mathrm{ml}$ of filtered PBS and size distribution was measured by Nanoparticle Tracking Analysis (Malvern Instruments Ltd., Malvern, UK). Then, MSCs-Exo were quantified by bicinchoninic acid (BCA) assay 
(Thermo Scientific) for measurement of total protein. MSCs-Exo were identified by the marker proteins CD63 using western blot. $1 \mu \mathrm{l}$ MSCs-Exo were dissolved in $100 \mu \mathrm{lPBS}$. The diluted MSCs-Exo were prelabeled by PKH26 (Sigma-Aldrich, San Louis, MO, US) and co-cultured for $12 \mathrm{~h}$ in the supernatant of fibroblasts that had reached $60 \%-70 \%$ confluence in the 24 -well plate.

\section{Isolation and culture of atrial fibroblasts}

Primary atrial fibroblasts were isolated from 1- to 3-day-old BALB/c mice as previously described. Fibroblasts were cultured in Dulbecco's Modified Eagle's Medium (DMEM)/F12 supplemented with 10\% fetal bovine serum and $1 \%$ penicillin-streptomycin in $37^{\circ} \mathrm{C}$ humidified incubator with $5 \% \mathrm{CO}_{2}$. Cells in passage 2-3 were used for in vitro study. Angiotensin II (Ang-II, $100 \mathrm{nM}) \square M S C s-E x o(50 \mu \mathrm{g} / \mathrm{ml})$ were used to treat fibroblasts as indicated.

\section{Culture of MSCs}

DMEM plus 10\% exosome-free FBS and 1\% streptomycin/penicillin was used for the culture of MSCs. For transfection of miR-148a-3p inhibitors (100 nM), MSCs were seeded in 6-well plates and transfected with lipofectamine 3000 (Thermo Scientific).

\section{Animals and treatments}

8-week-old male BALB/c mice were purchased from Pengyue Laboratory Animal Co, Ltd (Shandong, China). For in vivo study, all animals were anesthetized with $2 \%$ isoflurane inhalation before euthanization. 30 mice were randomized into three groups, namely the sham procedure group, Ang-II treatment group, and Ang-II and MSCs-Exo co-treatment (Ang-II + MSCs-Exo) group. An osmotic pump (ALZET 2004, Cupertino, CA, US) filled with Ang-II $(1.0 \mathrm{mg} / \mathrm{kg} / \mathrm{min})$ or PBS $(200 \mu \mathrm{l})$ were implanted subcutaneously after anesthesia. In the Ang-II + MSCs-Exo group, 300ug/150ul of MSCs-Exo were injected into tail veins a week before Ang-ll pump implantation and twice a week (every Monday and Thursday) for 28 days after establishing the experimental animal model.

\section{Ultrasonic cardiogram analysis}

On the 28th day post-Ang-II or saline infusion, echocardiography was performed with ultrasound instrument (Vivid 7, GE Healthcare). Mice were anesthetized with $2 \%$ isoflurane inhalation. The size of left atrium size was assessed by apical four-chamber view and was measured at end-ventricular systole from the tip of the mitral valve closure to the base of the LA $[10,11]$.

\section{Electrophysiological investigation for AF inducibility}

Programmed intracardiac stimulation was performed to evaluate AF inducibility as previously described $[12,13]$. A 1.1F electrode catheter (Science, Canada) was advanced into the right atrium through the right external jugular vein. A burst of programmed electrical stimulation was used to evaluate the inducibility of AF. Each burst stimulation lasted for $5 \mathrm{~s}$, starting from the cycle length of $50 \mathrm{~ms}$, decreasing in each 
successive burst by a $2 \mathrm{~ms}$ decrement to the cycle length of $10 \mathrm{~ms}$. Following these successive bursts, the incidence of $\mathrm{AF}$ and $\mathrm{AF}$ duration were analyzed. $\mathrm{AF}$ was defined as the occurrence of rapid and irregular atrial excitation lasting at least $1 \mathrm{~s}$, and the time from the end of burst pacing to the first $P$ wave was measured after rapid irregular atrial rhythm. The incidence of inducible AF was calculated as the percentage of the AF-inducible mice divided by the total number of mice.

\section{Histology}

The hearts were quickly removed under anesthesia.

The left atrium was fix with $4 \%$ formaldehyde after washing with PBS. Tissues were imbedded in paraffin and cut into slices of $5 \mathrm{~mm}$ thick. The slices were stained with Masson's trichrome staining to assess the degree of atrial fibrosis. Images were acquired and analyzed by Image-Pro Plus 6.0 software. In each atrium, five visual fields with a magnification of $100 \times$ and $200 \times$ were tested and analyzed.

\section{Immunofluorescence staining}

The atrial fibroblasts were seeded on confocal dish and were washed twice with PBS. The cells were fixed in $4 \%$ paraformaldehyde for $10 \mathrm{~min}$ at room temperature. Then, atrial fibroblasts were washed twice with PBS and permeabilized with $0.5 \%$ Triton X-100 for 20 min at room temperature. Cells were blocked with western blocking buffer (Beyotime, China) for $30 \mathrm{~min}$ at room temperature. The atrial fibroblasts were incubated with anti-a-SMA antibody (CST, 1:200) overnight at $4^{\circ} \mathrm{C}$. Subsequently, atrial fibroblasts were washed twice with PBS and incubated with Alexa Fluor 647 (Beyotime, 1:500, China) and were protected from light. Finally, the DNA was stained with DAPI, and images of the cells were captured using a Leica SP5 confocal microscope (Leica Microsystems, Mannheim, Germany).

\section{RNA isolation, PCR and quantitative real-time PCR}

Total RNA and miRNA were extracted from cultured atrial fibroblasts and left atrium using RNAeasy Mini Kit (Qiagen, Netherlands) and miRcute miRNA isolation kit (TIANGEN Biotech, China) according to the manufacturer's instructions. Further detection of miRNA and TGF- $\beta 1$ levels as well as RNA reverse transcription to cDNA using miRcute Plus miRNA First Strand cDNA Synthesis Kit (TIANGEN Biotech, China) and RevertAid First Strand Kit (Thermo Scientific, USA) were respectively performed according to the protocols. The primers of miR-148a-3p, TGF- $\beta 1, A L K 5$, U6 and GAPDH were designed and synthesized by Sangon Biotech Co., Ltd (Shanghai). The real-time PCR (RT-PCR) assay was performed with the ABI 7500 RT-PCR System (Applied Biosystems, CA, USA). Fold change of miRNA and mRNA were normalized with $U 6$ or GAPDH expression levels using the $2^{-\triangle \triangle C T}$ method. Primer sequences are listed in Supplemental Table 1.

\section{Public data mining to identify highly expressed microRNAs in MSCs-Exo}

We downloaded the two public MSCs-Exo miR abundance data from rat (PMID: 30715852) and from mouse (PMID: 30753344). For the first rat dataset, we first normalized the read count from multiple 
samples and log transformed the data. Then we selected the highly expressed miR by selected the miR with an expression level higher than the mean plus two standard deviation. For the second mouse study, the highly expressed miR list was obtained from their supplementary table directly with a TPM cutoff at 1000 .

\section{MiR-148a-3p targeted gene prediction and luciferase reporter gene assays}

We used TargetScan 7.2 (http://www.targetscan.org/) and miRDB (http://mirdb/org/) to predict and analyze the binding site of miR-148a-3p and ALK5 as well as that of miR-148a-3p and Smad2 as shown in Fig. 7. To test if ALK5 and Smad2 were the targets gene of miR-148a-3p, we separately constructed the 3'UTR of wild type ALK5 and Smad2, and then cloned them to the downstream pGL3 plasmid vector of the dual-luciferase reporter gene. Furthermore, we constructed the 3 'UTR of mutant type ALK5 and Smad2, which was replaced by the $6 \sim 7$ bp synthesized at the seed region of miR-148a-3p and then inserted in the same plasmid vector. HEK 293T cells at their logarithmic growth phase were collected and transferred to the 96 -well plate at $2 \times 10^{4}$ cells/well. The cells were incubated at $37^{\circ} \mathrm{C}$ and $5 \%$ of $\mathrm{CO}_{2}$ in a cultivation chamber. MiR-148a-3p mimics or inhibitor was transfected for $48 \mathrm{~h}$ according to the user's manual of Lipofectamine 3000 Transfection Reagent. The luciferase activity was tested with the dualluciferase reporter system (Promega, USA).

\section{Western blot analysis}

The mice left atrium tissues were lysed using RIPA lysis buffer (Beyotime Institute of Biotechnology, Shanghai, China) containing $1 \%$ protease inhibitor and phospholipase inhibitor. The protein concentration of each sample was quantified using BCA kit (BioRad, CA, USA). Briefly, electrophoretic separation was performed using a $12.5 \%$ SDS-polyacylamide gel and subsequently transferred to a PVDF membrane. Blocking was performed with 5\% nonfat milk in Tris-buffered saline with Tween 20 (TBST) for $1 \mathrm{~h}$ at room temperature. The antibodies for TGF- $\beta 1$, alpha-1 type I collagen (COL1a1), connective tissue growth factor (CTGF), Smad2, ALK5, p-ALK5 and GAPDH were purchased from Cell Signaling Technology (Danvers, USA). The membrane was exposed to an enhanced chemiluminescence kit (Millipore Corp, USA) and quantified using Image $J$ software.

\section{Statistical analysis}

Numerical data were expressed as mean \pm SEM. Two-tailed Student's $t$ test was used for data comparison of two groups with normal distribution, and one-way ANOVA followed by Holm-Sidak's test was used for multiple-group comparison with normal distribution. Mann-Whitney and Kruskal-Wallis test followed by Dunn's test were used to compare groups when the data were not normally distributed. Fisher's exact or Chi-squared tests were used to compare categorical data. A p-value $<0.05$ is considered statistically significant.

\section{Results}




\section{Characterization of exosomes derived from MSCs}

MSCs-Exo was morphologically similar with a typical cup-shaped structure, and the median size was 100 $\mathrm{nm}$ as visualized by TEM (Fig. 1A). Nanoparticle tracking analysis (NTA) showed that most MSCs-Exo were 100-110 nm in diameter (Fig. 1B). Western blot analysis showed that MSCs-Exo was positive for the exosome-specific marker CD63, further confirming the purity of the exosomes (Fig. 1C).

\section{MSCs-Exo attenuate Ang-II induced atrial fibrosis and atrial enlargement}

The process of experimental operation was shown in Fig. 2A. After continuous Ang-II infusion for 4 weeks, mice in Ang-II group showed marked atrial fibrosis compared with mice in Sham group. However, atrial fibrosis level was reduced significantly in the Ang-II + MSCs-Exo group compared with the Ang-II group (Fig. 2B-C). Moreover, western blot analysis showed that the protein expressions of TGF- $\beta 1$, COL1a1 and CTGF in the Ang-II group increased significantly compared with the Sham group, while their expressions decreased significantly in the Ang-II+MSCs-Exo group (Fig. 2D-F).

Echocardiogram was used to evaluate cardiac structure and function. Parameters of echocardiogram were summarized in Table 1. At the 28th day post Ang-ll pump implantation, atrial enlargement and ventricular hypertrophy can be seen as evidenced by increased left atrial diameter (LAD), end-diastolic ventricular septal thickness (IVSd), end-diastolic left ventricular posterior wall thickness (LVPWd), decreased left ventricular end-diastolic inner diameter (LVIDd) and left ventricular end-systolic inner diameter (LVIDs). 4 weeks-treatment with MSCs-Exo can significantly improve the Ang-II-induced left atrial enlargement and interventricular septum thickening, while the left ventricular ejection fraction (LVEF) and fractional shortening (FS) were not significantly different among these groups (Table 1).

\section{MSCs-Exo attenuate Ang-II induced AF inducibility}

Electrocardiograms were recorded in mice of each group, and their parameters of electrocardiography were summarized in Table 2. The P wave duration, PR interval as well as the corrected QT interval increased in mice post Ang-II infusion compared to that post saline infusion. However, compared with the Ang-II group, the increasing trend in such indicators reduced significantly in the Ang-II + MSCs-Exo group (Table 2). As we known, severe atrial fibrosis has been associated with an increased inducibility of AF. To verify whether aggravated atrial fibrosis post Ang-II infusion led to the lower threshold for AF inducibility, we performed programed electrophysiological study as mentioned previously. In the whole electrophysiological study, none of mice showed spontaneous arrhythmia. Compared with mice post saline infusion, inducibility of AF and AF duration increased in mice post Ang-II infusion. Treatment with MSCs-Exo significantly decreased Ang-II-induced AF inducibility and the AF duration (Fig. 3).

\section{MSCs-Exo ameliorate Ang-II-induced proliferation, phenotypic switching and secretion functions of fibroblasts}

For the sake of verifying the effects of MSCs-Exo on proliferation, phenotypic switching and secretion functions of fibroblasts, Ang-II was used to stimulate atrial fibroblasts (FBs) to trans-differentiate into 
myofibroblasts (MFBs) [14]. The differentiation was confirmed using immunofluorescence staining for aSMA, a specific marker for MFBs. After MSCs-Exo intervention, the amount of a-SMA fluorescence expression was reduced, indicating that MSCs-Exo inhibited the expression of a-SMA in the cells (Fig. 4A). Subsequently, to further define whether MSCs-Exo can be endocytosed by MFBs, MSCs-Exo was labeled with the fluorescent dye PKH26. After incubating for 2 hours, red fluorescence signals were detected in MFBs over time (Fig. 4B). By12 h, nearly all MFBs exhibited red fluorescence. The fluorescence intensity of a-SMA decreased with higher intake of exosomes, suggesting the upregulated a-SMA expression post Ang-II stimulation was inhibited by MSCs-Exo treatment. These finding demonstrate that MSCs-Exo are readily taken up by MFBs. Thus, exosomes can be used therapeutically to modulate FBs function. Moreover, CCK-8 assay showed that treatment with different concentrations of MSCs-Exo $(10 \mu \mathrm{g} / \mathrm{ml}, 50$ $\mu \mathrm{g} / \mathrm{ml}$ and $100 \mu \mathrm{g} / \mathrm{ml}$ ) did not affect the cell viability of MFBs (Fig. 4C). We used CCK-8 assay to assess the proliferation of the FBs. We found that Ang-II stimulation for $48 \mathrm{~h}$ showed elevated proliferation of FBs, however, treatment with $50 \mathrm{\mu g} / \mathrm{ml}$ MSCs-Exo could exhibited less proliferation after Ang-II stimulation (Fig. 4D). Thus the MSCs-Exo concentration of $50 \mu \mathrm{g} / \mathrm{ml}$ was used for subsequent experiments.

\section{Data mining of public available data on miRNA in MSCs-derived exosomes}

Exosomes regulated large numbers of physiological activities via exosomal miRNAs. So we explored whether MSCs-Exo was involved in regulating Ang-II-induced atrial fibrosis and vulnerability to AF through exosomal miRNAs. We obtained the overlapped list of highly expressed miRs from two studies (Fig. 5A). Then we selected the top five most abundant miRs based on the study from the shared list, performed additional literature search for these candidates to examine whether their reported functions were consistent with phenotype we observed before. Finally, we narrowed to two miR candidates: miR-148a-3p (ranked 1st and 2nd) and miR-24-3p (ranked 3rd and 29th ) (Fig. 5B).

\section{Effect of MSCs-Exo on proliferation, phenotypic switching and secretion functions of fibroblasts is mediated through miR-148a-3p}

Using RT-PCR, we confirmed that miR-148a-3p showed higher expression levels in MSCs-Exo (Fig. 6A). Interestingly, stimulating primary cultured fibroblasts with Ang-ll significantly reduced the level of miR148a-3p, however, MSCs-Exo treatment remarkably increased the level of miR-148a-3p but not miR-24-3p (Fig. 6B). Therefore, we concluded that miR-148a-3p might play a crucial role in the effects of MSCs-Exo on Ang-Il-induced atrial fibrosis and vulnerability to AF and only focused on miR-148a-3p for the further functional studies. To confirm the role of miR-148a-3p in MSCs-Exo, we inhibited the function of miR148a-3p in MSCs-Exo by transfecting MSCs with $100 \mathrm{nM}$ miR-148a-3p inhibitors and then isolated the exosomes (called miR-148a-3p inhibitor MSCs-Exo) from the culture supernatants. RT-PCR demonstrated that the level of miR-148a-3p was significantly reduced in miR-148a-3p inhibitor MSCs-Exo compared with negative control (NC) inhibitor MSCs-Exo (Fig. 6C). We then treated Ang-ll-stimulated fibroblasts with miR-148a-3p inhibitor MSCs-Exo for $48 \mathrm{~h}$ and then collected the cells for CCK-8 and RT-PCR analysis. Results showed that the alleviated proliferation and downregulation of fibrosis-related genes expression 
with MSCs-Exo were partially negated by miR-148a-3p inhibitor MSCs-Exo (Fig. 6D-E). These findings suggested that miR-148a-3p recapitulated the effects of MSCs-Exo, while inhibiting miR-148a-3p blunted those effects. MiR-148a-3p was involved in the alleviation of MSCs-Exo on Ang-Il-induced atrial fibrosis and vulnerability to AF.

\section{MiR-148a-3p shuttling by MSCs-Exo modulated Ang-II-induced atrial fibrosis and vulnerability to atrial fibrillation through targeting ALK5 and Smad2}

To better understand how miR-148a-3p modulated Ang-II-induced atrial fibrosis and vulnerability to AF, we focused on the target genes of miR-148a-3p. 577 and 697 potential target genes for miR-148a-3p were predicted respectively using TargetScan and miRDB with 344 common ones (Fig. 7A). Pathway enrichment analysis using the Kyoto Encyclopedia of Genes and Genomes (KEGG) identified the TGF- $\beta 1$ signalling pathway as a potential target pathway through which miR-148a-3p regulates fibrosis, with ALK5 and Smad2 being predicted targets of miR-148a-3p. TargetScan predicted that both the position 1607-1635 of ALK5 3' UTR and the position 8334-8340 of SMAD2 3' UTR were potential binding sites of miR-148a-3p. We constructed dual luciferase reporter plasmids pGL3-ALK5-3' UTR, pGL3-SMAD2-3' UTR, pGL3-ALK5-3' UTR-mutant and pGL3-SMAD2-3' UTR-mutant (Fig. 7B). Dual luciferase reporter assay showed that miR-148a-3p mimic inhibited and miR-148a-3p inhibitor enhanced reporter luciferase activity of ALK5 and Smad2. The above changes were abolished after mutating the binding sites (Fig. 7C). These results suggested that miR-148a-3p could inhibit the expression of ALK5 and Smad2.

Previous studies demonstrated that TGF- $\beta 1$, the ligand of ALK5, can be induced by Ang-II and played an important role via the classical Smad2/3 pathway in the development of atrial fibrosis. We further investigated the effects of MSCs-Exo on TGF- $31 /$ ALK5/ Smad2 signaling pathway. We found that the protein expressions of TGF- $\beta 1$, ALK5, p-ALK5, COL1a1 and Smad2 were significantly downregulated in fibroblasts after MSCs-Exo treatment. The inhibition of TGF- $\beta 1 / A L K 5 /$ Smad2 signaling pathway was partially reversed when treated with miR-148a-3p inhibitor MSCs-Exo (Fig. 7D). These results suggested that miR-148a-3p was involved in the MSCs-Exo mediated atrial fibrosis and AF inducibility by targeting TGF-B1/ALK5/ Smad2 signaling pathway (Fig. 8).

\section{Discussion}

As a novel cell-free therapeutic approach [15], MSCs-Exo has been reported to be therapeutically efficacious in a variety of diseases including heart [16], liver [17], kidney [18], immune and neurological diseases [19]. As for the cardiovascular diseases, MSCs-Exo exert its cardioprotective effects by promoting angiogenesis [20], suppressing inflammation [21] and cardiomyocyte apoptosis [22] in infarcted heart [23], attenuating myocardial ischemia/reperfusion injury [24]. However, the role of MSCsExo in the pathogenesis of atrial fibrosis and AF inducibility remains unknown. In our study, we firstly demonstrated the therapeutic effects of MSCs-Exo on Ang-II induced atrial fibrosis and vulnerability to AF. Our work further confirmed that miR-148a-3p was abundant in MSCs-Exo, and regulated cellular property of fibroblasts via targeting ALK5/Smad2 axis. 
As we known, atrial fibrosis is the hallmark of AF structural remodeling, and it is also associated with AF recurrences and resistance to therapy. Here, in Ang-II induced atrial fibrosis mice model, MSCs-Exo alleviated atrial fibrosis and AF inducibility. Atrial fibrosis is characterized by abnormal proliferation of atrial fibroblasts and excessive deposition of extracellular matrix [25]. In vitro studies showed that in AngII-treated fibroblasts, MSCs-Exo reduced Ang-II-induced fibroblasts proliferation, phenotypic switching, cellular migration and collagen production. All these results indicated the anti-fibrotic role of MSCs-Exo in AF.

MiRNAs make up a key fraction of components of MSCs-Exo [26]. Among the miRNAs found in MSCsExo, some miRNAs have the anti-fibrotic property, including miR-214-3p [27], miR-21 [28], miR-23a [25], miR-125b [29], miR-145 [30] and so on. To reveal the molecular mechanisms responsible for the observed findings, we identified miR-148a-3p as candidate by combing the data mining of public available datasets on MSCs-Exo and functional validations. Until now, previous studies have suggested that as a member of miR-148/-152 family, miR-148a-3p was involved in the biology of cancer [31] and maintenance of epigenetic patterns [32], regulating the inflammatory response [33] and liver fibrosis. However, studies concerning miR-148a-3p and fibrosis are limited, and the role of miR-148a-3p especially derived from MSCs-Exo in the pathogenesis of cardiac fibrosis remains unknown. Our study first revealed that blocking miR-148a-3p in MSCs-Exo blunted the alleviated effects of MSCs-Exo on atrial fibrosis and AF inducibility.

Previous studies suggested that miR-148a-3p targeted ERBB3 to regulate alcoholic liver fibrosis and the viability of hepatic stellate cells [34]. Moreover, miR-148a-3p targeted Smad2 to regulate the invasion and metastasis of cancer [35]. TGF- $\beta 1 / \mathrm{Smad} 2 / 3$ pathway plays a key role in matrix remodeling of atrium, capable of promoting cellular proliferation and migration, activating fibroblasts differentiation into myofibroblasts, and accelerating extracellular matrix synthesis, which are associated with atrial fibrosis and vulnerability to AF. Several studies have demonstrated that TGF- $\beta 1$ had a profibrotic effect via the classical Smad signaling pathway, which potentiated atrial fibrosis and AF. Significantly, whether miR148a-3p or TGF- $\beta 1 /$ ALK5/Smad2 pathway was involved in MSCs-Exo-mediated alleviation of atrial fibrosis was explored by our group. The results in our study firstly revealed that miR-148a-3p enriched in MSCs-Exo targeted the two key proteins in the TGF- $\beta 1$ signaling pathway, namely ALK5 and Smad2. Furthermore, we found that Ang-II-stimulated TGF- $\beta 1 /$ ALK5/Smad2 signaling pathway was inhibited after MSCs-Exo treatment, and the inhibition of TGF-B1/ALK5/ Smad2 signaling pathway was partially reversed when treated with miR-148a-3p inhibitor MSCs-Exo. These data strongly indicated the involvement of miR-148a-3p in MSCs-Exo-mediated atrial fibrosis and AF inducibility by targeting TGF$\beta 1 / A L K 5 / S m a d 2$ pathway. Admittedly, picking a single miRNA candidate might oversimplify the actual biology. Though our study suggested that exosomal miR-148a-3p played an important role on the antifibrotic effects of MSCs-Exo, we could not eliminate the contribution of other exosomal cargoes.

\section{Conclusions}


In conclusion, our study demonstrated that MSCs-Exo improved Ang-ll induced atrial fibrosis and AF inducibility. MiR-148a-3p packaged in MSCs-Exo was involved in the above-mentioned process by targeting ALK5/Smad2 axis.

\section{Abbreviations}

AF: Atrial fibrillation; MSCs: Mesenchymal stem cells; MSCs-Exo: MSCs-derived exosome; TEM : Transmission electron microscopy; Ang-ll: Angiotensin-Il; Ang-II+MSCs-Exo: Ang-Il and exosome treatment; BCA: Bicinchoninic acid; DMEM: Dulbecco's Modified Eagle's Medium; FBs: Fibroblasts; MFBs: Myofibroblasts; CTGF: Connective tissue growth factor; LAD: Left atrial diameter; IVSd: End-diastolic ventricular septal thickness; LVPWd: End-diastolic left ventricular posterior wall thickness; LVIDd: Left ventricular end-diastolic inner diameter; LVEF: Left ventricular ejection fraction; FS: Fractional shortening; COL1a1: Alpha-1 type I collagen; NTA: Nanoparticle tracking analysis (NTA).

\section{Declarations}

\section{Acknowledgements}

The authors wished to thank Mr. Baoxiang Zhuang at the Morphology Laboratory and Prof. Zhaodong Juan at the Shandong Provincial Medicine and Health Key Laboratory of Clinical Anesthesia of Weifang Medical University for their invaluable assistances in Immunohistochemical studies.

\section{Authors' contributions}

Daoliang Zhang and Qijuan Sun conceived the original idea and supervised the project. Jie Liu performed the experiments and analyzed the data. Qian Wang wrote and revised the manuscript with help from Liye Zhang and Rui Zhang. Shili Jiang performed the IHC analysis. Wenbo Liu, Huirong Han and Guangchen Zou edited the manuscript. All authors read and approved the final manuscript.

\section{Funding}

This work was supported by Shanghai Sailing Program (19YF1431800), National Natural Science Foundation of China (81900293), Shandong Medical and Health Technology Development Program (2019WS595), Natural Science Foundation of Shandong (ZR2017MH066), Nurture projects for basic research of Shanghai Chest Hospital (2018YNJCM03), Interdisciplinary Program of Shanghai Jiao Tong University (ZH2018QNB30), Research project of Traditional Chinese Medicine of Shanghai Municipal Health and Family Planning Commission (2018JP009).

\section{Availability of data and materials}

All data generated and analyzed during this study are included in this published article.

\section{Ethical statement}


All experimental procedures were performed with the approval of the Ethics Committee as well as the Animal Care and Use Committee of Shanghai Chest Hospital affiliated to Shanghai Jiao Tong University.

\section{Consent for publication}

Not applicable.

\section{Competing interests}

The authors declare that the research was conducted in the absence of any commercial or financial relationships and there are no competing interests.

\section{References}

1. Nattel S, Burstein B, Dobrev D. Atrial remodeling and atrial fibrillation: mechanisms and implications. Circ Arrhythm Electrophysiol. 2008;1:62-73. Doi: 10.1161/circep.107.754564.

2. Nattel S. Molecular and cellular mechanisms of atrial fibrosis in atrial fibrillation. JACC Clin Electrophysiol. 2017;3:425-435. Doi: 10.1016/j.jacep.2017.03.002.

3. Phinney DG, Pittenger MF. Concise review: MSC-derived exosomes for cell-free Therapy. Stem Cells. 2017;35:851-858. Doi: 10.1002/stem.2575.

4. Zhao J, Li X, Hu J, Chen F, Qi, et al. Mesenchymal stromal cell-derived exosomes attenuate myocardial ischaemia-reperfusion injury through miR-182-regulated macrophage polarization. Cardiovasc Res. 2019;115:1205-1216. Doi: 10.1093/cvr/cvz040.

5. Li Y, Yang R, Guo B, et al . Exosomal miR-301 derived from mesenchymal stem cells protects myocardial infarction by inhibiting myocardial autophagy. Biochem Biophys Res Commun. 2019;514:323-328. Doi:10.1016/j.bbrc.2019.04.138.

6. O'Brien K, Breyne K, Ughetto S, et al. RNA delivery by extracellular vesicles in mammalian cells and its applications. Nat Rev Mol Cell Biol. 2020;21:585-606. Doi:10.1038/ s41580-020-0251-y.

7. Kore RA, Wang X, Ding Z, et al. MSC exosome-mediated cardioprotection in ischemic mouse heart comparative proteomics of infarct and peri-infarct areas. Mol Cell Biochem. 2021;476:1691-1704. Doi: 10.1007/s11010-020-04029-6.

8. Zhu LP, Tian T, Wang JY, et al. Hypoxia-elicited mesenchymal stem cell-derived exosomes facilitates cardiac repair through miR-125b-mediated prevention of cell death in myocardial infarction. Theranostics. 2018;8:6163-6177. Doi: 10.7150/thno.28021.

9. Bang C, Batkai S, Dangwal S, et al. Cardiac fibroblast-derived microRNA passenger strand-enriched exosomes mediate cardiomyocyte hypertrophy. J Clin Invest. 2014;124:2136-46. Doi: 10.1172/jci70577.

10. Granillo A, Pena CA, Pham T, et al. Murine echocardiography of left atrium, aorta, and pulmonary artery. J Vis Exp. 2017;120:1-11. Doi:10.3791/55214. 
11. Medrano G, Hermosillo-Rodriguez J, Pham T, et al. Left atrial volume and pulmonary artery diameter are noninvasive measures of age-related diastolic dysfunction in mice. J Gerontol A Biol Sci Med Sci. 2016;71:1141-1150. Doi: 10.1093/gerona/glv143.

12. Yao C, Veleva T, Scott L, Jr. et al. Enhanced cardiomyocyte NLRP3 inflammasome signaling promotes atrial fibrillation. Circulation. 2018;138:2227-2242. Doi:

10.1161/circulationaha.118.035202.

13. Wang Q, Yu Y, Zhang P, et al. The crucial role of activin A/ALK4 pathway in the pathogenesis of AngIl-induced atrial fibrosis and vulnerability to atrial fibrillation. Basic Res Cardiol. 2017;112(47):1-17. Doi: 10.1007/s00395-017-0634-1.

14. Li S, Gao Y, Liu Y, et al. Myofibroblast-derived exosomes contribute to development of a susceptible substrate for atrial fibrillation. Cardiology. 2020;145:324-332. Doi: 10.1159/000505641

15. Jafarinia M, Alsahebfosoul F, Salehi $H$, et al. Mesenchymal stem cell-derived extracellular vesicles: A novel cell-free therapy. Immunol Invest. 2020;49:758-780. Doi:10.1080/08820139.2020.1712416.

16. Kim H, Yun N, Mun D, et al. Cardiac-specific delivery by cardiac tissue-targeting peptide-expressing exosomes. Biochemical and Biophysical Research Communications. 2018;499:803-808. Doi: 10.1016/j.bbrc.2018.03.227.

17. Song XJ, Zhang L, Li Q, et al. hUCB-MSC derived exosomal miR-124 promotes rat liver regeneration after partial hepatectomy via downregulating Foxg1. Life Sci. 2021;265:1-9. Doi: 10.1016/j.lfs.2020.118821.

18. Ferguson CM, Farahani RA, Zhu XY, et al. Mesenchymal stem/stromal cell-derived extracellular vesicles elicit better preservation of the intra-renal microvasculature than renal revascularization in pigs with renovascular disease. Cells. 2021;10:1-16. Doi: 10.3390/cells10040763.

19. Reza-Zaldivar EE, Hernandez-Sapiens MA, Minjarez B, et al. Potential effects of MSC-derived exosomes in neuroplasticity in Alzheimer's disease. Front Cell Neurosci. 2018;12:1-16. Doi: 10.3389/fncel.2018.00317.

20. Liang $X$, Zhang $L$, Wang $S$, et al. Exosomes secreted by mesenchymal stem cells promote endothelial cell angiogenesis by transferring miR-125a. J Cell Sci. 2016;129:2182-2189. Doi:10.1242/jcs.170373.

21. Sun X, Shan A, Wei Z, et al. Intravenous mesenchymal stem cell-derived exosomes ameliorate myocardial inflammation in the dilated cardiomyopathy. Biochem Biophys Res Commun. 2018;503:2611-2618. Doi: 10.1016/j.bbrc.2018.08.012.

22. Liu $Z, X u Y, W a n Y$, et al. Exosomes from adipose-derived mesenchymal stem cells prevent cardiomyocyte apoptosis induced by oxidative stress. Cell Death Discov. 2019;5:1-7. Doi: 10.1038/s41420-019-0159-5.

23. Zhang Z, Yang J, Yan W, et al. Pretreatment of cardiac stem cells with exosomes derived from mesenchymal stem cells enhances myocardial repair. J Am Heart Assoc. 2016;5:1-16. Doi: 10.1161/jaha.115.002856.

24. Wei Z, Qiao S, Zhao J, et al. miRNA-181a over-expression in mesenchymal stem cell-derived exosomes influenced inflammatory response after myocardial ischemia-reperfusion injury. Life 
Sciences. 2019;232:1-11. Doi: 10.1016/j.Ifs.2019.116632.

25. Li J, Zhang Q and Jiao H. LncRNA NRON promotes M2 macrophage polarization and alleviates atrial fibrosis through suppressing exosomal miR-23a derived from atrial myocytes. J Formos Med Assoc. 2021;120:1512-1519. Doi:10.1016/j.jfma.2020.11.004.

26. Kalluri R and LeBleu VS. The biology, function, and biomedical applications of exosomes. Science. 2020;367(eaau6977):1-15. Doi: 10.1126/science.aau6977.

27. Lei $X, \mathrm{He} \mathrm{N}$, Zhu L, et al. Mesenchymal stem cell-derived extracellular vesicles attenuate radiationinduced lung injury via miRNA-214-3p. Antioxid Redox Signal. 2020;33(2):1-46. Doi: 10.1089/ars.2019.7965.

28. Qiao L, Hu S, Liu S, et al. MicroRNA-21-5p dysregulation in exosomes derived from heart failure patients impairs regenerative potential. J Clin Invest. 2019;129(6):2237-2250.

Doi:10.1172/JCl123135.

29. Nagpal V, Rai R, Place AT, et al. MiR-125b is critical for fibroblast-to-myofibroblast transition and cardiac fibrosis. Circulation. 2016;133:291-301. Doi: 10.1161/circulationaha.115.018174.

30. Fang S, Xu C, Zhang Y, et al. Umbilical cord-derived mesenchymal stem cell-derived exosomal microRNAs suppress myofibroblast differentiation by inhibiting the transforming growth factorB/SMAD2 pathway during wound healing. Stem Cells Trans/ Med. 2016;5:1425-1439. Doi: 10.5 66/sctm.2015-0367.

31. Jung KH, Zhang J, Zhou C, et al. Differentiation therapy for hepatocellular carcinoma: Multifaceted effects of miR-148a on tumor growth and phenotype and liver fibrosis. Hepatology. 2016;63:864-879. Doi: 10.1002/hep.28367.

32. Friedrich $M$, Pracht $K$, Mashreghi MF, et al. The role of the miR-148/-152 family in physiology and disease. Eur J Immunol. 2017;47:2026-2038.Doi: 10.1002/eji.201747132.

33. Fang $Y, X u X Y$, Shen $Y$, et al. MiR-148 targets CiGadd45ba and CiGadd45bb to modulate the inflammatory response to bacterial infection in grass carp. Dev Comp Immunol. 2020;106:103611. Doi: 10.1016/j.dci.2020.103611.

34. Xiong J, Ni J, Chen C, et al. MiR-148a-3p regulates alcoholic liver fibrosis through targeting ERBB3. Int J Mol Med. 2020;46:1003-1012. Doi:10.3892/ijmm.2020.4655.

35. Wu Y, Gu W, Han X, et al. LncRNA PVT1 promotes the progression of ovarian cancer by activating TGF- $\beta$ pathway via miR-148a-3p/AG01 axis. J Cell Mol Med. 2021;00:1-15. Doi: 10.1111/jcmm. 16700 .

\section{Tables}


Table 1

Parameters of echocardiography in mice of Sham group, Ang-II group and Ang-II + MSCs-Exo group

\begin{tabular}{|llll|}
\hline & Sham & Ang-II & Ang-II + MSCs-Exo \\
\hline LAD, mm & $2.8 \pm 0.5$ & $4.5 \pm 0.4^{* *}$ & $3.0 \pm 0.5^{\# \#}$ \\
\hline IVSd, mm & $0.7 \pm 0.1$ & $1.0 \pm 0.1^{* *}$ & $0.8 \pm 0.1^{\# \#}$ \\
\hline LVPWd, mm & $0.7 \pm 0.1$ & $0.9 \pm 0.1^{* *}$ & $0.7 \pm 0.1^{\# \#}$ \\
\hline LVIDd, mm & $3.8 \pm 0.1$ & $3.2 \pm 0.3^{* *}$ & $3.7 \pm 0.2^{\# \#}$ \\
\hline LVIDs, mm & $1.9 \pm 0.1$ & $1.7 \pm 0.1^{*}$ & $1.8 \pm 0.1^{\# \#}$ \\
\hline EF, \% & $63.8 \pm 6.5$ & $33.8 \pm 1.5$ & $33.5 \pm 1.6$ \\
\hline FS, \% & $35.5 \pm 1.5$ & \\
\hline All values are expressed as means \pm SEM, N = 6 in each group. & \\
\hline *p<0.05, **p<0.01 vs Sham group; \#p<0.05, \#\#p<0.01 vs Ang-II group. \\
\hline $\begin{array}{l}\text { LAD, left atrial diameter; IVSd, Interventricular septal end-diastolic thickness; LVPWd, LV end-diastolic } \\
\text { posterior wall thickness; LVEDV, LV end-diastolic volume; LVESV, LV end-systolic volume; LVEF, LV } \\
\text { ejection fraction; LVFS, LV fractional shortening. }\end{array}$ \\
\hline
\end{tabular}

Table 2

Parameters of electrocardiography in mice of Sham group, Ang-ll group and Ang-II+MSCs-Exo group

\begin{tabular}{|c|c|c|c|}
\hline & Sham & Ang-II & Ang-II + MSCs-Exo \\
\hline HR, bpm & $445.8 \pm 20.1$ & $440.3 \pm 17.4$ & $452.2 \pm 15.8$ \\
\hline $\mathrm{P}, \mathrm{ms}$ & $11.6 \pm 1.0$ & $22.2 \pm 2.8^{\star *}$ & $15.7 \pm 1.5^{\# \#}$ \\
\hline $\mathrm{PR}, \mathrm{ms}$ & $40.2 \pm 3.2$ & $56.0 \pm 4.7^{\star \star}$ & $43.0 \pm 3.0^{\# \#}$ \\
\hline QT, ms & $23.2 \pm 2.1$ & $39.3 \pm 3.1^{\star *}$ & $27.7 \pm 3.6^{\# \#}$ \\
\hline \multicolumn{4}{|c|}{ All values are expressed as means $\pm S E M, N=6$ in each group. } \\
\hline \multicolumn{4}{|c|}{${ }^{*} p<0.05,{ }^{* *} p<0.01$ vs Sham group; $\# p<0.05, \# \# p<0.01$ vs Ang-II group } \\
\hline HR, heart & e; bpm, beats & minute. & \\
\hline
\end{tabular}

\section{Figures}


A

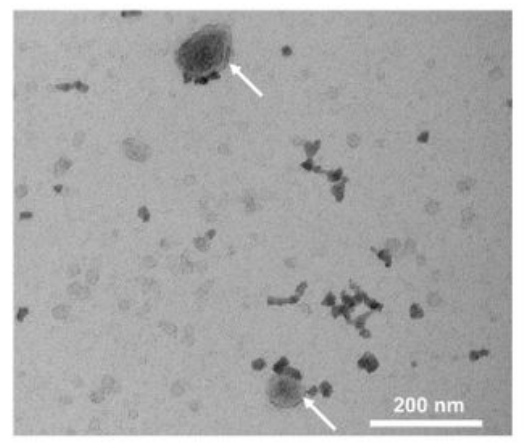

B

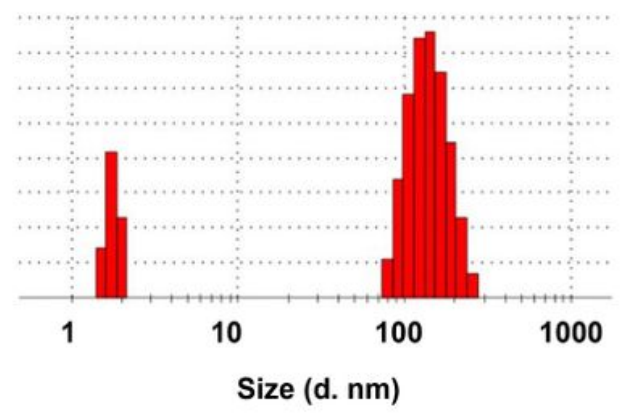

C

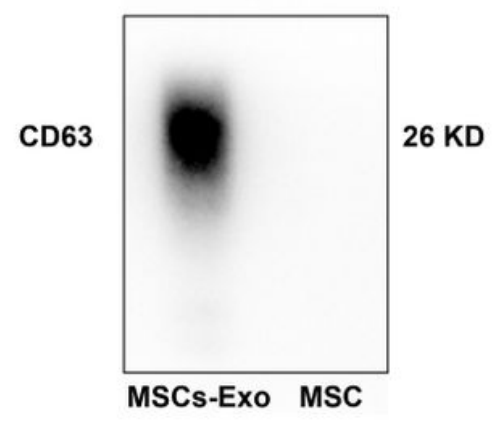

Figure 1

Characterization and functional validation of exosomes derived from mesenchymal stem cells (MSCsExo). (A) Cup-shaped morphology of purified exosomes assessed by TEM. Scale bar $=200 \mathrm{~nm}$. (B) NTA analysis of MSCs-Exo shows that exosomes had the typical size range $(30 \sim 120 \mathrm{~nm})$. (C) Exosomal markers CD63 assessed by western blot. 
A

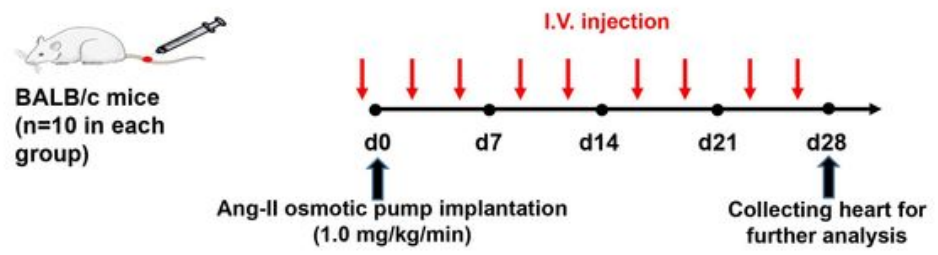

B
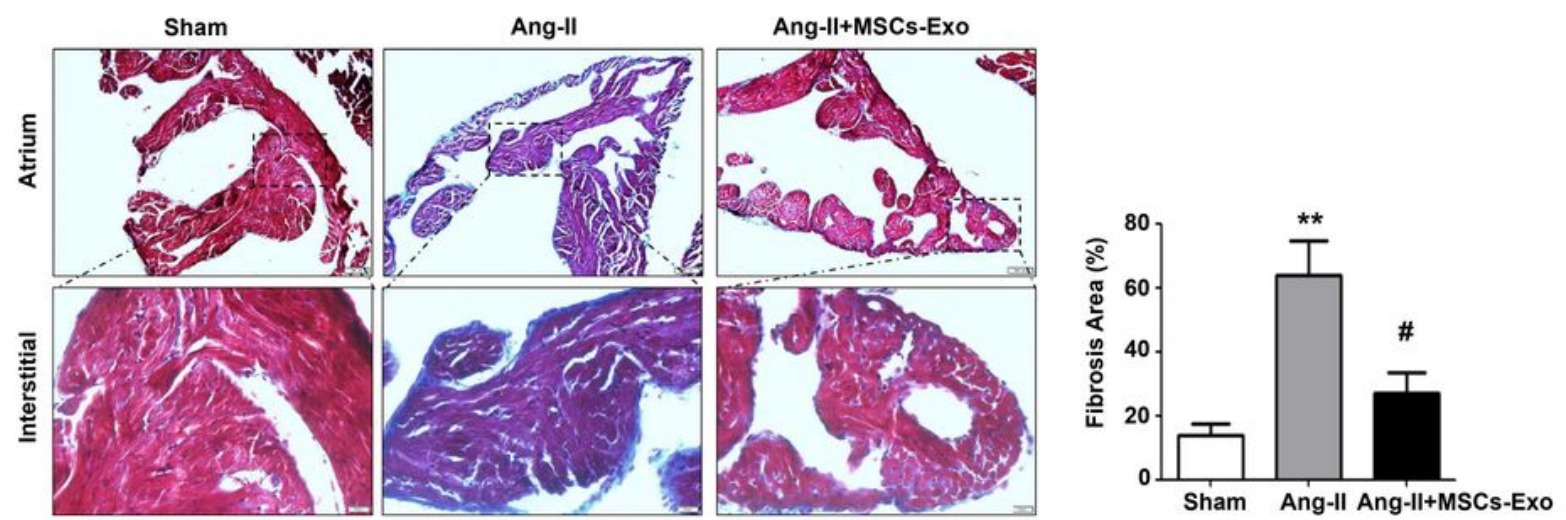

C
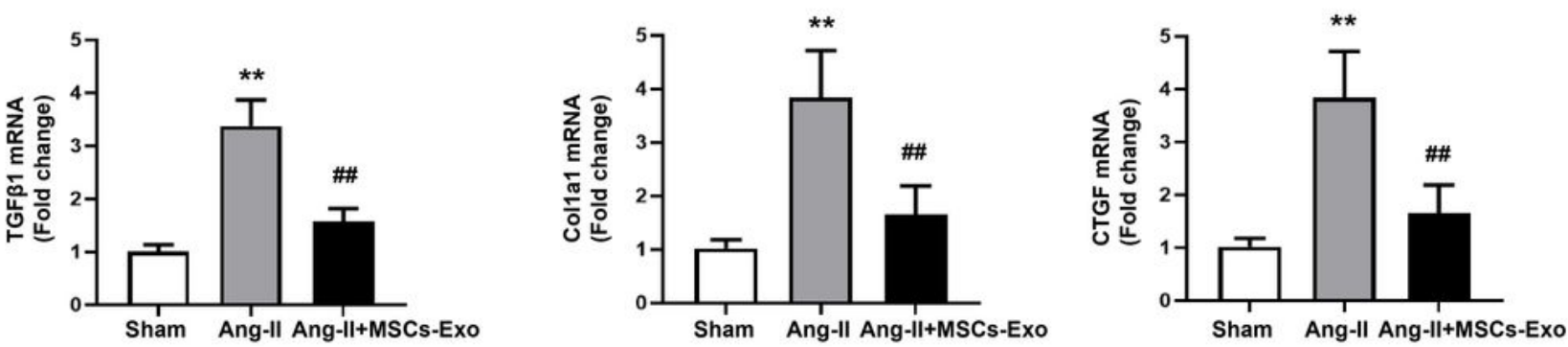

D
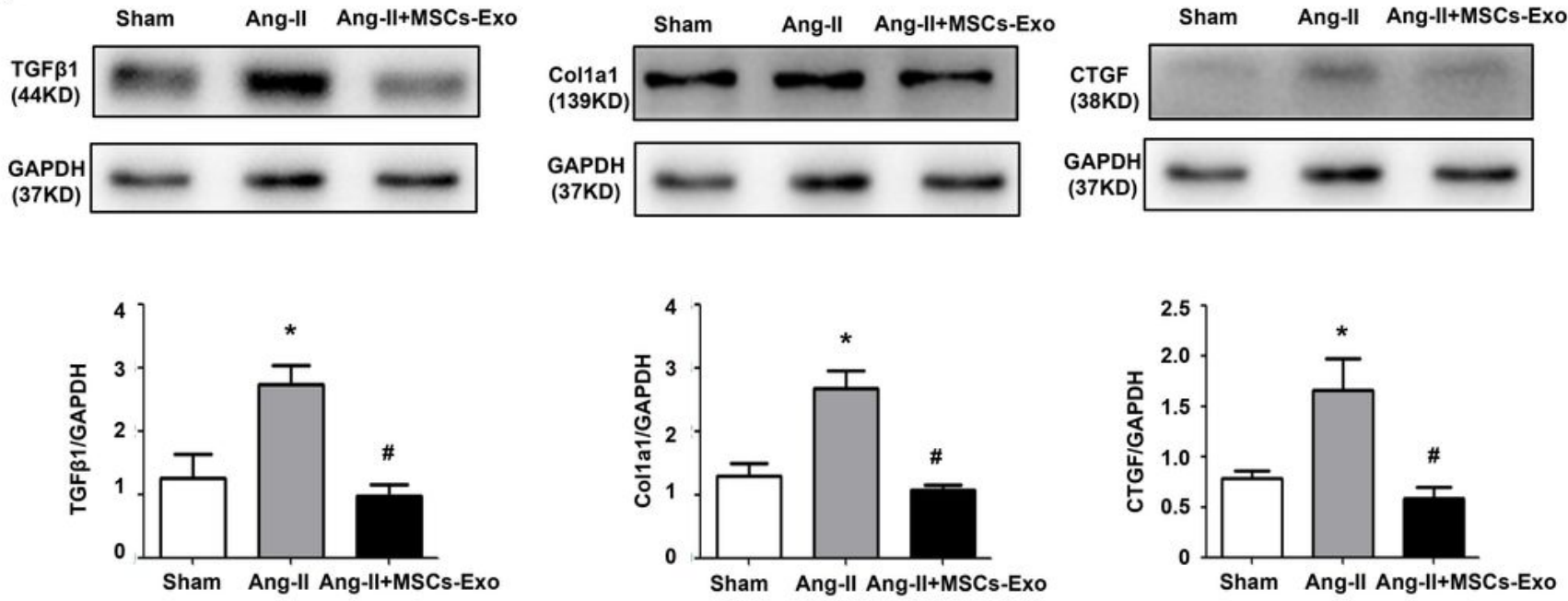

Figure 2

MSCs-Exo attenuate Ang-Il-induced atrial fibrosis. (A) Schematic description of the experimental design. $300 \mu \mathrm{g} / 150 \mathrm{ul}$ of MSCs-Exo were injected into tail veins a week before Ang-Il pump implantation and twice a week (every Monday and Thursday) for 28 days after establishing the experimental animal model. (B) Masson's trichrome staining of hearts from sham and Ang-II treated mice and Ang-II+MSCs-Exo treated mice. Scale bar= $100 \mu \mathrm{m}$. (C) Quantification of interstitial fibrosis by calculating collagen volume fraction 
3 weeks after operation. ( $n=5)$. (D) Western blot analysis shows the protein expression levels of TGF- $\beta 1$, COL1a1 and CTGF in atria tissues from sham and Ang-II treated mice and and Ang-II+MSCs-Exo treated mice. Data were presented as mean \pm SEM. $N=3$ in each group. ${ }^{*} P<0.05$ vs Sham group, ${ }^{\star *} \mathrm{P}<0.01$ vs Sham group; \#P $<0.05$ vs Ang-Il group, \#\#P $<0.01$ vs Ang-II group.
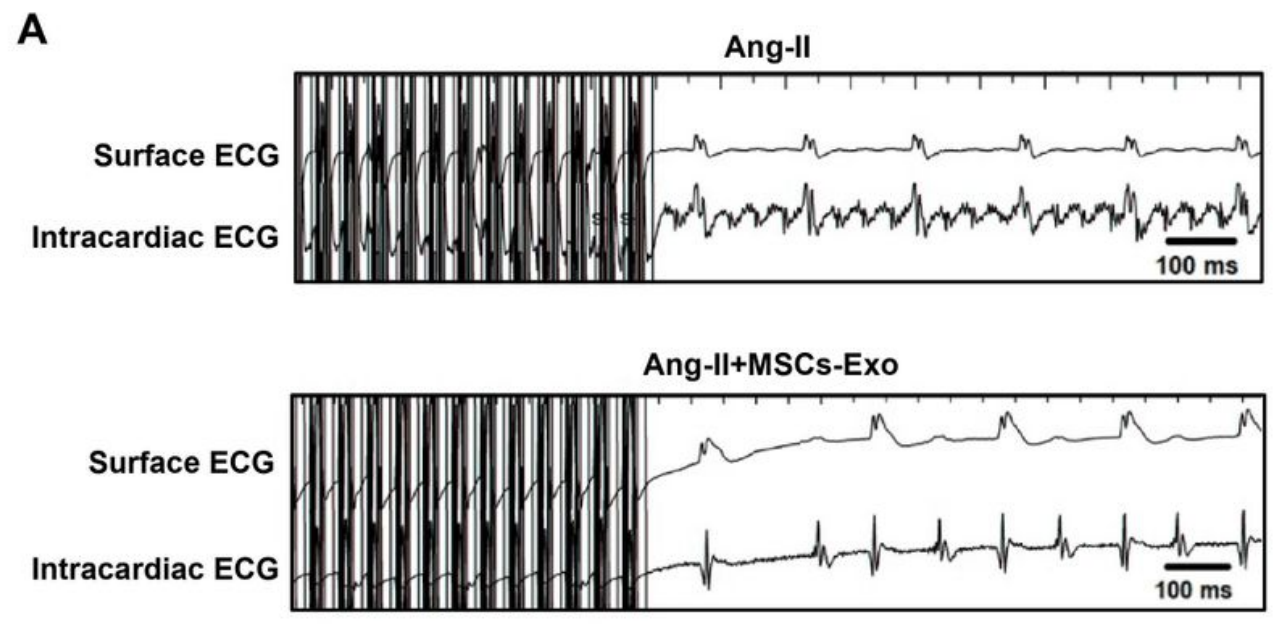

B

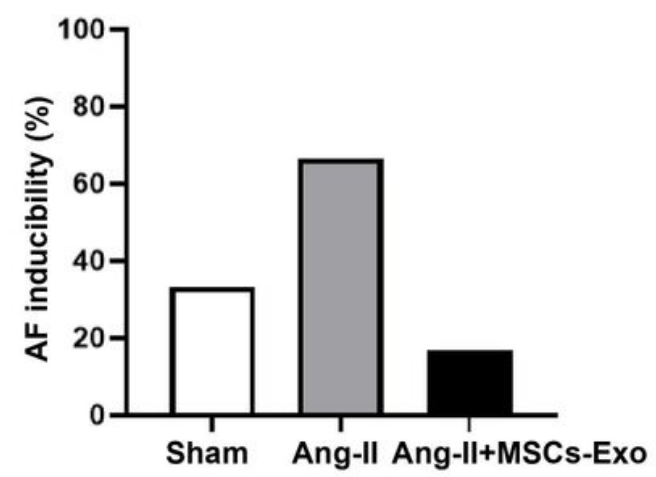

C

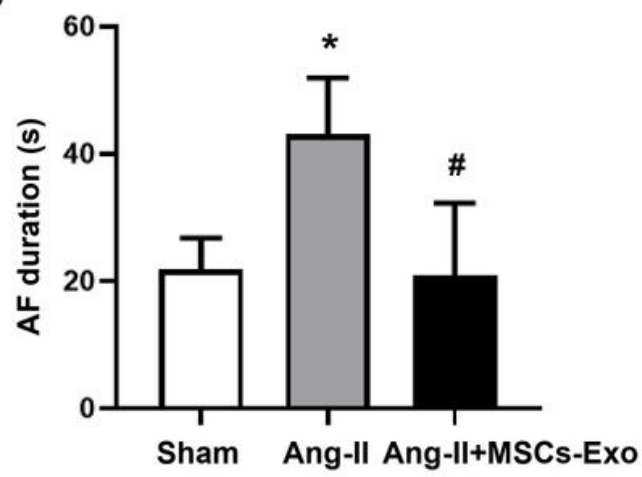

Figure 3

Treatment with MSCs-Exo significantly decreased Ang-II-induced AF inducibility and the AF duration. (A) Representative surface and intracardiac ECG tracings of Sham, Ang-II treated mice and Ang-II+MSCs-Exo treated mice during electrophysiological studies with the burst pacing protocol (cycle length $=30 \mathrm{~ms}$, scale $=100 \mathrm{~ms}$ ). (B) AF inducibility and AF durations in the indicated groups during the electrophysiological studies. Data were presented as mean \pm SEM. $N=6$ in each group. ${ }^{*} P<0.05$ vs Sham group, ${ }^{\star \star} \mathrm{P}<0.01$ vs Sham group; \#P $<0.05$ vs Ang-II group, \#\#P $<0.01$ vs Ang-Il group. 


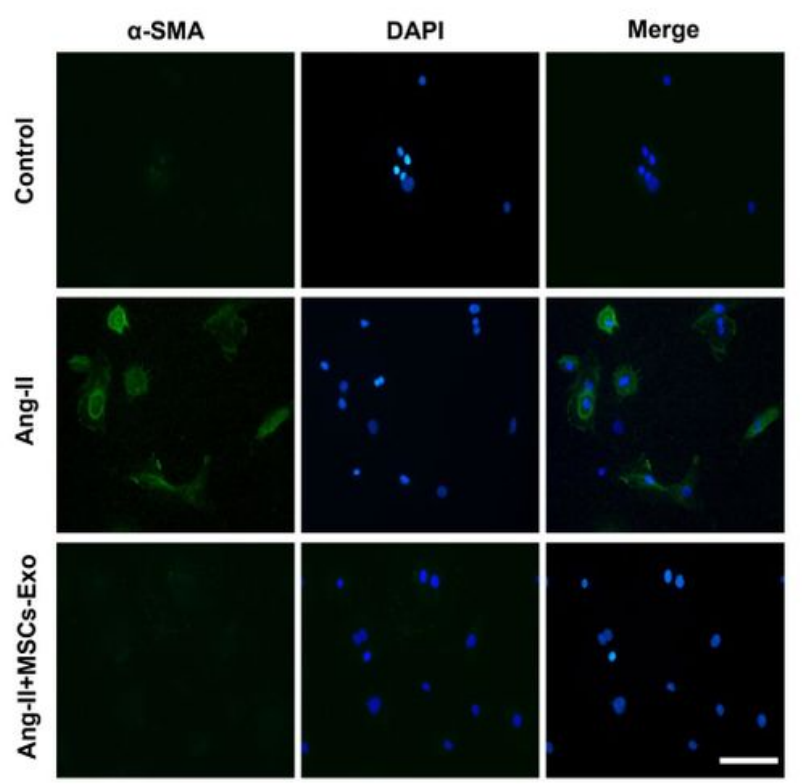

E

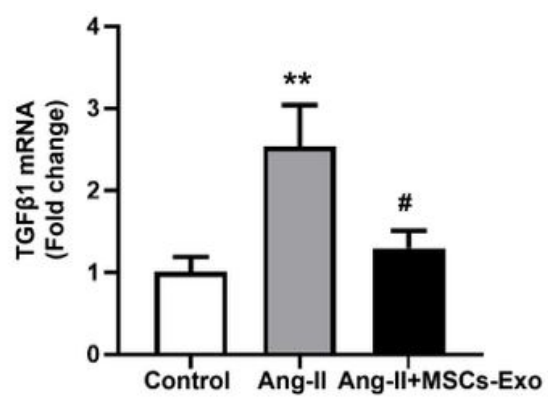

B

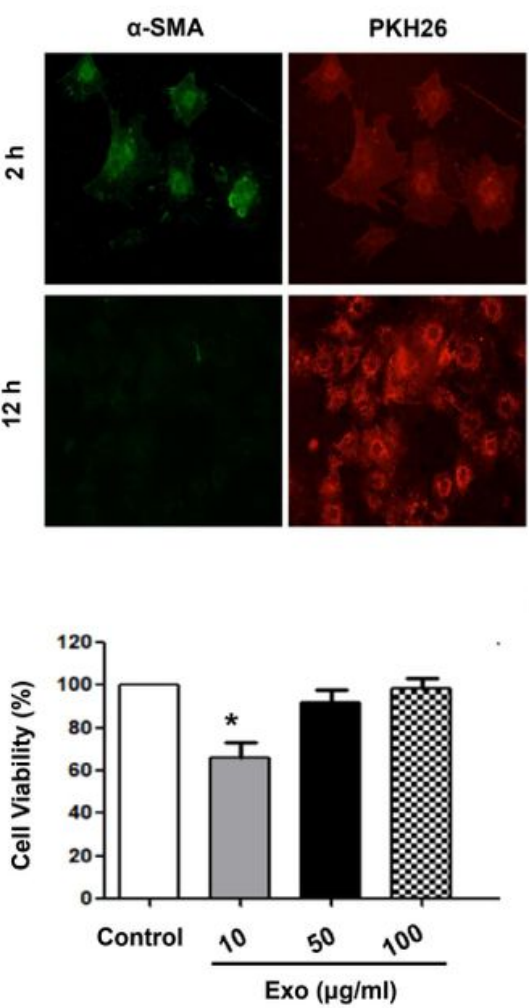

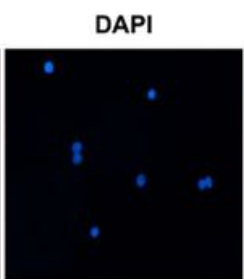

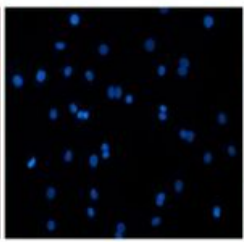

D

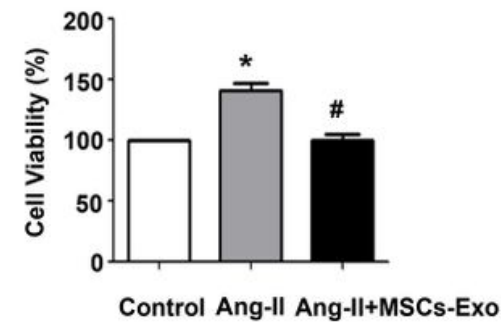

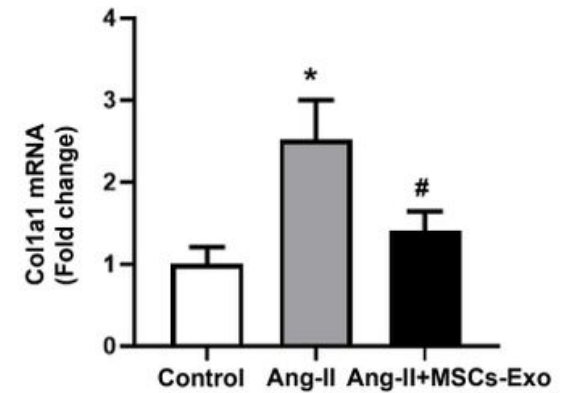

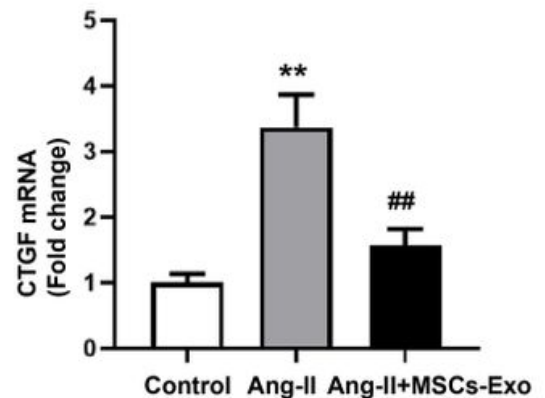

Figure 4

MSCs-Exo ameliorate Ang-II-induced proliferation, phenotypic switching and secretion functions of fibroblasts. Treatment with Ang II induces activation of FBs into MFBs. FBs were treated with Ang-II (100 nM) for 24 h. (A) Untreated FBs (Control), Ang Il-treated MFBs and Ang-II+MSCs-Exo treated MFBs were stained with a-SMA (green) and DAPI (blue). (B) Fluorescence microscopy detection of the uptake of PKH26-labeled MSCs-Exo (red) by MFBs (a-SMA green, DAPI blue) in $2 \mathrm{~h}$ and $12 \mathrm{~h}$. Scale bar $=100 \mu \mathrm{m}$. (C) Cell viability treated with PBS (Control) or dedicated concentrations of MSCs-Exo $(10 \mu \mathrm{g} / \mathrm{ml}, 50 \mu \mathrm{g} / \mathrm{ml}$, or $100 \mu \mathrm{g} / \mathrm{ml}$ ). (D) Cell viability treated with PBS (Control), Ang II (100 nM) and Ang-Il+MSCs-Exo (50 $\mu \mathrm{g} / \mathrm{ml})$. (E) Ang-ll-induced mRNA expressions of fibrosis-related genes were assessed by RT-PCR in each group. Data were presented as mean \pm SEM. $N=3$ in each group. ${ }^{*} \mathrm{P}<0.05$ vs Control group, ${ }^{*} \mathrm{P}<0.01$ vs Control group; \#P $<0.05$ vs Ang-II group, \#\#P $<0.01$ vs Ang-Il group. 
A

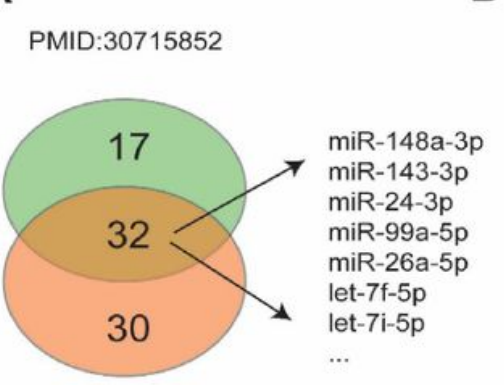

PMID:30753344

B

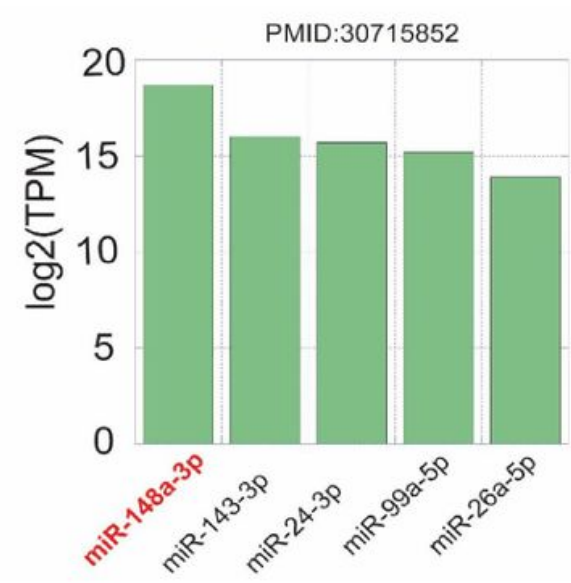

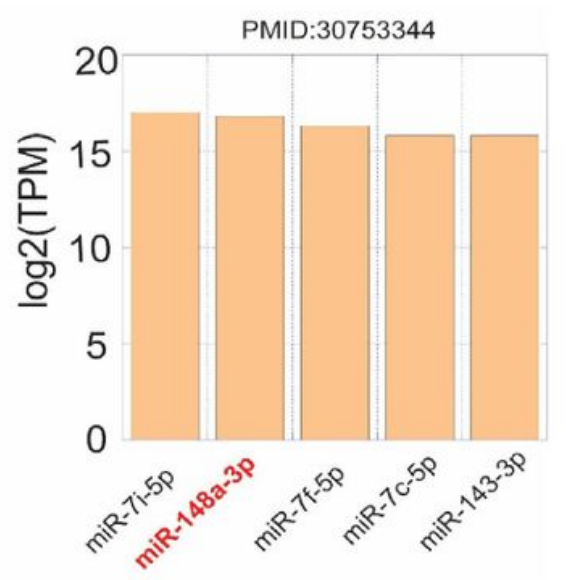

\section{Figure 5}

Data mining of public available data on miRNA in MSCs-derived exosomes. (A) Overlapped list of highly expressed miRs from PMID 30715852 and PMID 30753344. (B) Select the top five most abundant miRs based on the study from the shared list. 
A

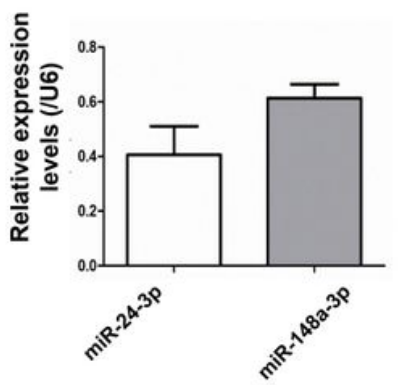

D

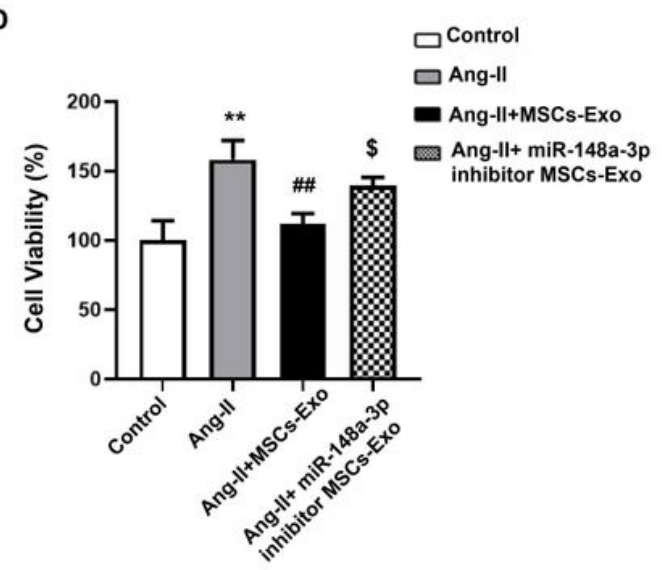

B

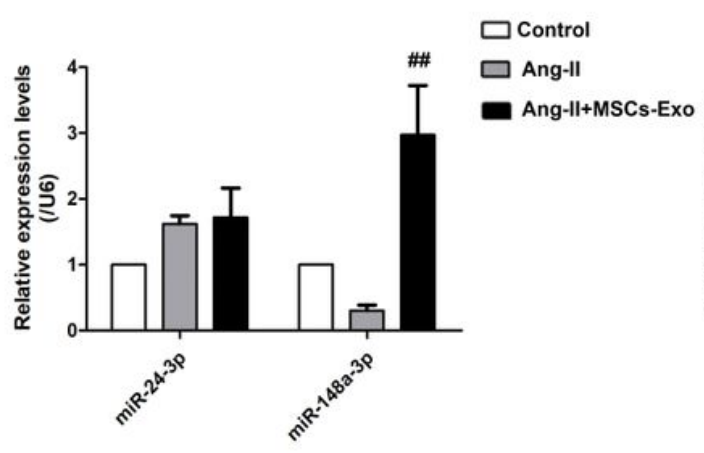

$E$

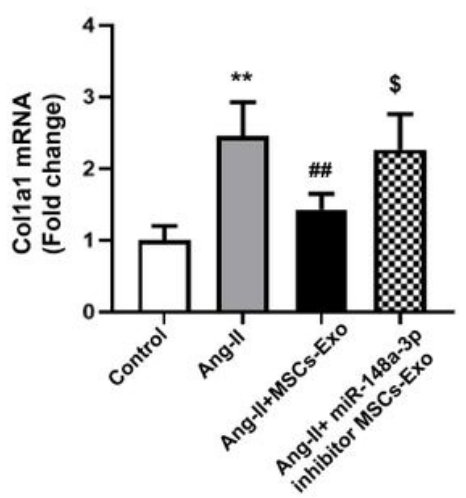

C $\square$ Control

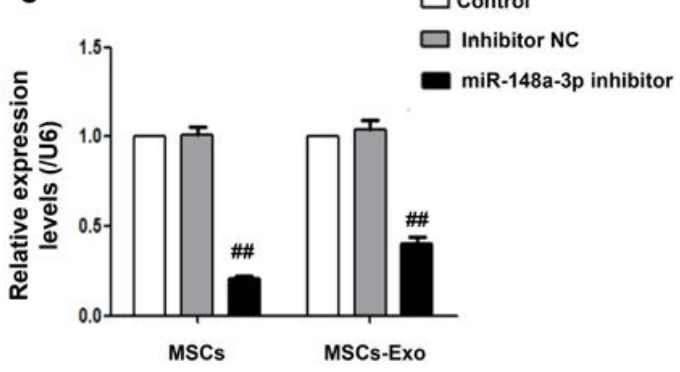

$\square$ Control

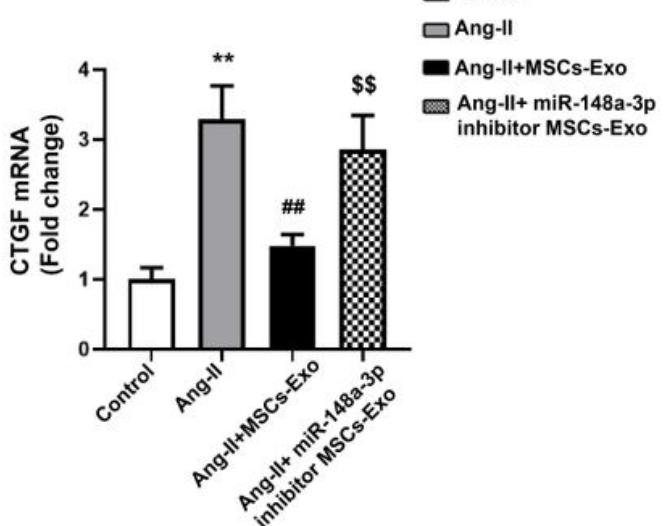

\section{Figure 6}

Effects of MSCs-Exo on proliferation, phenotypic switching and secretion functions of fibroblasts are mediated through miR-148a-3p. (A) RT-PCR analysis of miR-24-3p and miR-148a-3p levels in MSCs-Exo. Data were normalized to U6. (B) The levels of miR-24-3p and miR-148a-3p in untreated FBs (Control), Ang II-treated MFBs and Ang-II+MSCs-Exo treated MFBs after 24h. (C) miR-148a-3p levels in MSCs and MSCsExo transfected by negative control (NC) inhibitor or miR-148a-3p inhibitor. (D) Cell viability treated with PBS (Control), Ang II (100 nM), Ang-II+MSCs-Exo (50 $\mathrm{gg} / \mathrm{ml})$ and Ang-ll+miR-148a-3p inhibitor MSCs-Exo $(50 \mu \mathrm{g} / \mathrm{ml})$. (E) Ang-II-induced mRNA expressions of fibrosis-related genes were assessed by RT-PCR in each group. Data were presented as mean \pm SEM. $N=3$ in each group. ${ }^{*} \mathrm{P}<0.05$ vs Control group, $* \star P<$ 0.01 vs Control group; \#P $<0.05$ vs Ang-ll group, \#\#P $<0.01$ vs Ang-ll group; $\$ \mathrm{P}<0.05$ vs Ang-II+MSCsExo group, $\$ \$ P<0.01$ vs Ang-II+MSCs-Exo group. 
A

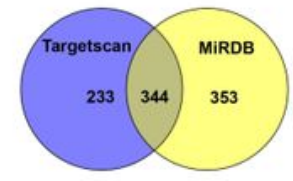

B

pGL3-SMAD2-3' UTR-mutant $\quad$ 5'-GGCUGCAUCCUUGGACGUGACU-3'

PGL3-SMAD2-3' UTR 5'-GGCUGCAUCCUUGGUGCACUGU-3' IIIIIII

hsa-miR-148a-3p 3'-UGUUUCAAGACAUCACGUGACU-5' IIIIII

PGL3-ALK5-3' UTR 5'-GAACACCCCACAGGAGCACUGG-3'

PGL3-ALK5-3' UTR-mutant 5'-GAACACCCCACAGGACGUGACG-3'

C
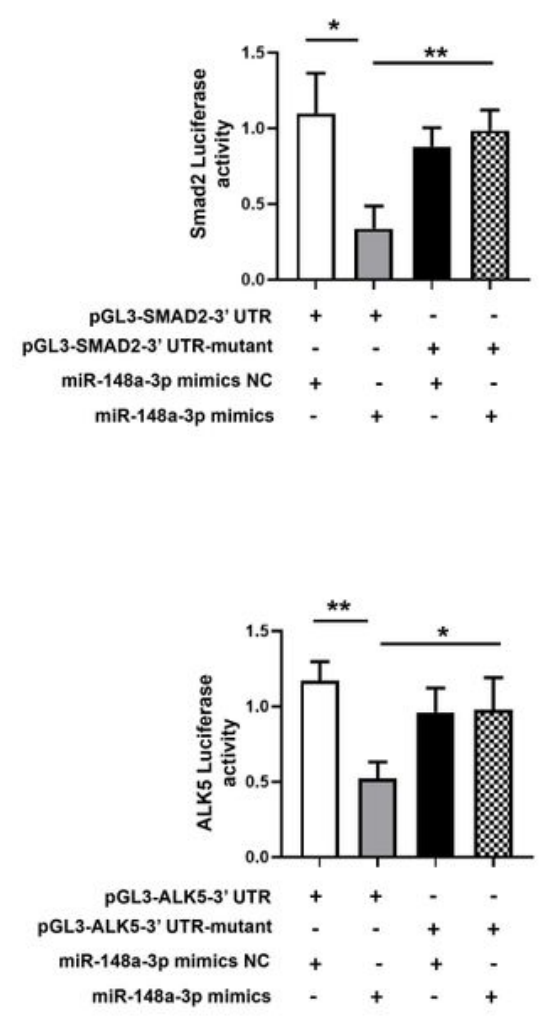
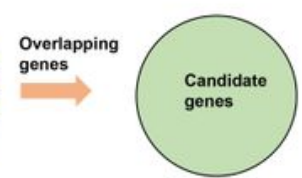

Literature

search

ALK5 Smad2

D

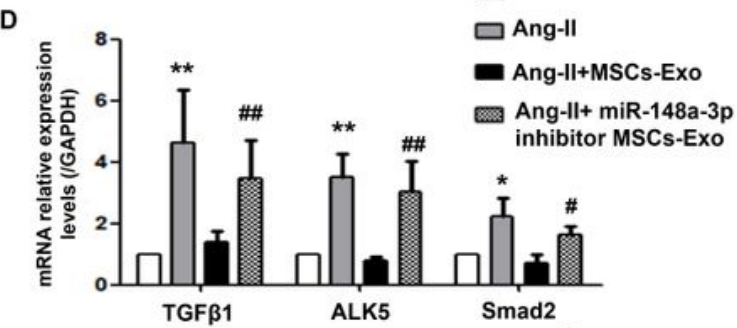

E

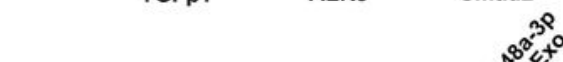

$\square$ Control

$\square$ Ang-ll

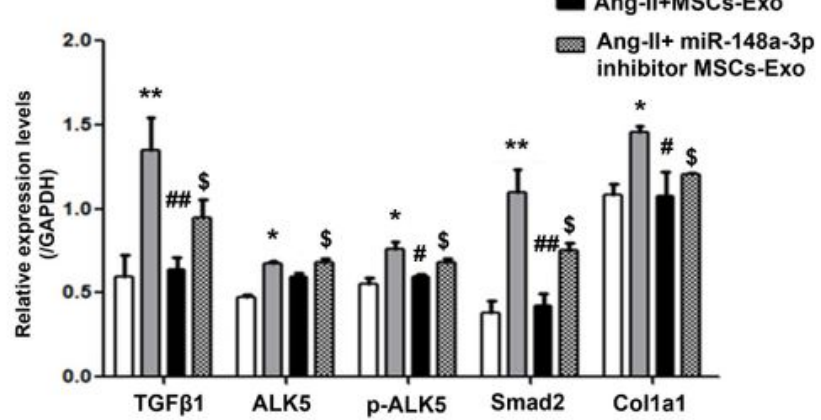

\section{Figure 7}

MiR-148a-3p targeted ALK5/Smad2 axis and inhibited the expression of TGF- $31 / A L K 5 / S m a d 2$ pathway. (A) Flowchart for determining the target genes of miR-148a-3p. (B) Schematic image of binding site between miR-148a-3p and SMAD2-3'UTR as well as that between miR-148a-3p and ALK5-3'UTR. (C) The HEK 293T cells were co-transfected with pGL3-SMAD2-3'UTR, pGL3-SMAD2-3'UTR-mutant, pGL3-ALK53'UTR, pGL3-ALK5-3'UTR-mutant or miR-148a-3p mimics. 48 h post transfection, the indicated cells were 
subjected to dual luciferase assay. (D) The mRNA expressions of TGF- $\beta 1 / A L K 5 /$ Smad2 pathway in untreated FBs (Control), Ang II-treated MFBs, Ang II+MSCs-Exo treated MFBs and Ang II+ miR-148a-3p inhibitor MSCs-Exo treated MFBs by RT-PCR. (E) Western blots analysis for the protein levels of TGF- $\beta 1$, ALK5, p-ALK5, COL1a1 and Smad2 in untreated FBs (Control), Ang II-treated MFBs, Ang II+MSCs-Exo treated MFBs and Ang II+ miR-148a-3p inhibitor MSCs-Exo treated MFBs for 48h. Data were presented as mean \pm SEM. $N=3$ in each group. ${ }^{*} \mathrm{P}<0.05$ vs Control group, ${ }^{*} \mathrm{P}<0.01$ vs Control group; $\# \mathrm{P}<0.05$ vs Ang-II group, \#\#P $<0.01$ vs Ang-II group; $\$ \mathrm{P}<0.05$ vs Ang-II+MSCs-Exo group, $\$ \$ \mathrm{P}<0.01$ vs Ang-II+MSCsExo group.

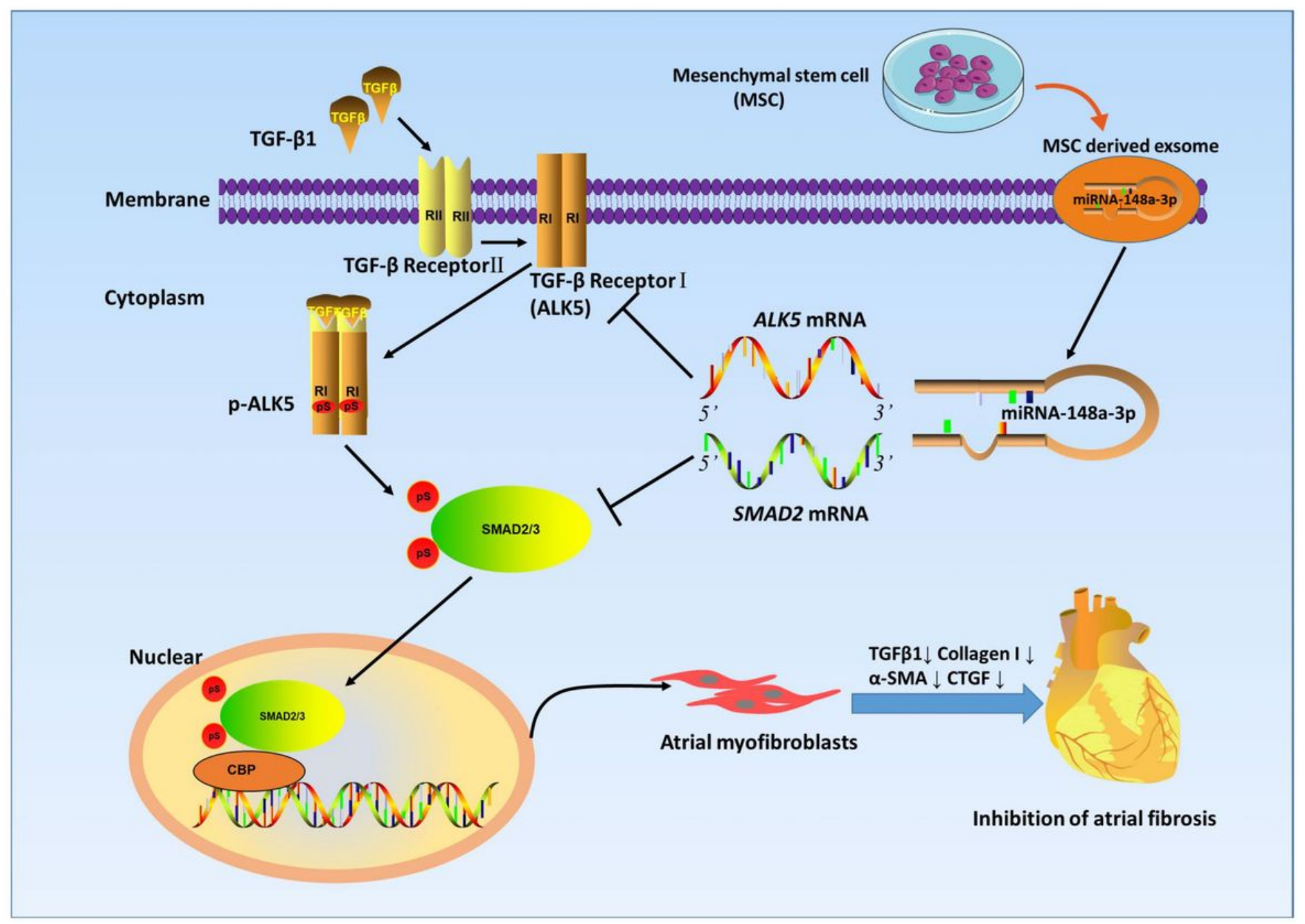

\section{Figure 8}

A schematic representation of the current study. MSCs-Exo could effectively alleviate Ang-II induced atrial fibrosis and vulnerability to AF. miR-148a-3p was identified to be abundant in MSCs-Exo and played an important role on the anti-fibrotic effects by targeting ALK5/Smad2 axis.

\section{Supplementary Files}

This is a list of supplementary files associated with this preprint. Click to download. 
- SUPPLEMENTALTABLES.docx

Page 25/25 